\title{
Fabrication and adhesion of hierarchical micro-seta
}

\author{
ZHANG Hao $^{1}$, WU LianWei ${ }^{1}$, JIA ShiXing $^{2}$, GUO DongJie $^{1^{*}} \&$ DAI ZhenDong ${ }^{1}$ \\ ${ }^{1}$ Institute of Bio-inspired Structure and Surface Engineering, Nanjing University of Aeronautics \& Astronautics, Nanjing 210016, China; \\ ${ }^{2}$ The 55th Research Institute of Ministry of the Electronics Industry, Nanjing 210016, China
}

Received December 21, 2011; accepted February 16, 2012

\begin{abstract}
Spark-erosion perforating technology was used to fabricate a Cu-based template characterized by pores with radius of $0.5 \mathrm{~mm}$ inclined at $75^{\circ}$. A commercial silicone elastomer of poly(dimethylsiloxane) (PDMS) with a rich Si-H content was used to produce an inclined array of primary setae. The technique of argon ion plasma etching on crystalline silicon was used to fabricate negative templates with radii of 5, 10, and $20 \mu \mathrm{m}$. The Si-H rich PDMS was used to cast three types of fine array templates, which acted as the secondary setae. A vinyl-rich PDMS precursor was used to bind the primary and secondary setae by a hydrosilylation reaction, thus allowing the formation of three different hierarchical arrangements of setae. Adhesion tests demonstrated that shear adhesion was anisotropic, first increasing in strength then decreasing to a stable level as slippage occurred. The adhesion strength was significantly influenced by the nature of the secondary setae, showing a strong correlation with aspect-ratio and concentration.
\end{abstract}

seta, poly(dimethylsiloxane), adhesion, hierarchical structure

Citation: Zhang H, Wu L W, Jia S X, et al. Fabrication and adhesion of hierarchical micro-seta. Chin Sci Bull, 2012, 57: 1343-1349, doi: 10.1007/s11434-0125076-4

The gecko is an excellent model to assist in the design of a three-dimensional obstacle-free (TDOF) robot with high locomotory performance for inspection and fault detecting with potential for military applications [1]. A proposed specification for such a robot is that loaded with communication equipment with $5 \mathrm{~km}$ working range $(10 \mathrm{~g})$, a $0.3 \mathrm{~mol}$ $\mathrm{L}^{-1}$ pixel optical sensor $(10 \mathrm{~g})$, a power source $(40-50 \mathrm{~g})$, the gecko can climb ten floors within $20 \mathrm{~s}$. This climbing ability is derived from the structure of the feet and toes, which have millions of setae with hierarchical branched structures [2-5]. These micro-scale setae control contact between the toe and the surface, by van der Waals' intermolecular attractive forces.

This type of complex hierarchical structure also has adhesive [6] and anti-fouling properties [7]. Therefore, fabricating artificial micro setae has been the subject of much biomimetic research. Sitti et al. [8-12] fabricated negative pore templates by lithography and cast fine polyurethane (PU) setae, then implanted them onto a large radius setae surface, thus forming a double-level setae array. Strong

*Corresponding author (email: djguo@nuaa.edu.cn) bonding was achieved, but subsequent detachment of the setae was not possible. Jeong et al. [13-16] fabricated a high-aspect-ratio seta array by nano-injection molding, and the resultant setae array exhibited strong adhesion on a coarse textured surface. The angle of inclination of the negative pores could be tuned. Campo et al. [17,18] fabricated photo-resist (SU-8)-based templates with varying radii by the duplicated lithography method, then cast hierarchical poly(dimethylsiloxane) (PDMS) setae. The primary columnar setae had a height of $200 \mu \mathrm{m}$ and a radius of $25 \mu \mathrm{m}$, the secondary columnar setae had a height of $5 \mu \mathrm{m}$ and radii of $5,10,15 \mu \mathrm{m}$. Northen et al. [19-21] fabricated hydrophilic and hydrophobic setae arrays by plasma etching. Zhang et al. [22] cast photo-resist setae from a negative PDMS template with various depths by nano-imprinting. However, the photo-resist is easily damaged and has weak adhesion properties, limiting practical applications. Kustandi et al. [23] fabricated poly(methylmethacrylate) (PMMA) setae by using a negative template made from anodic aluminum oxide (AAO), which exhibited upright pores with varying pore radii between $50-500 \mu \mathrm{m}$. In China, Jiang et al. [24], Sun et al. [25], Wang et al. [26], and Sun et al. [27-32], have also 
been working in this area.

Based on structural and functional biomimic, we aim to fabricate gecko-inspired setae arrays with inclined hierarchical structures. Spark-erosion perforating technology was used to fabricate the inclined sub-millimeter-scale $\mathrm{Cu}$-based template for the primary setae; Argon ion plasma etching was used to fabricate the micrometer scale Si-based template for the secondary setae. A hydrosilylation reaction between the primary and secondary setae arrays was used to prepare three types of hierarchical micro-setae arrays. A tribometer (CETR, USA) with high sensitivity force sensor was used to measure adhesion. The gecko uses a "slipping adhesion" technique to achieve a high level of adhesion performance [2], where the adhesion of the setae arrays are strongly influenced by direction of motion, angle, and preload [33,34], this slipping adhesion is significantly affected by motion directions and preload. The aim of this paper is to fabricate a gecko-inspired micro-setae array for use in a TDOF robot.

\section{Materials and methods}

\subsection{Templates and materials}

A Cu-based template was made using spark-erosion perforating technology: the inclined linear array was obtained by angling the wire-electrode at $45^{\circ}$ to the planar $\mathrm{Cu}$ surface. The negative pore pattern (radius $0.5 \mathrm{~mm}$ ) was then perforated by spark-erosion inside the convex linear array. The crystalline Si templates were etched by argon ion plasma etching (Alcatel 601E, France) to a nominal pore depth of $30 \mu \mathrm{m}$. The crystalline Si had p-type $\left(\begin{array}{lll}1 & 0 & 0\end{array}\right)$ orientation with an electrical resistivity of $0.01 \Omega \mathrm{cm}$. The radii of the designed mask were 5,10 , and $15 \mu \mathrm{m}$, with pitches of 20,30 , and $30 \mu \mathrm{m}$, respectively. PDMS (Sylgard 184) was obtained from Dow Corning (USA). PU (PX223HT) was obtained from Axson (France). Other reagents were reagent grade and were used without further purification.

\subsection{Fabricating the primary setae}

A uniform primary seta array was prepared based on an optimization of former techniques [29]. The PU precursor (mass ratio of 1:1) was poured into a polytetrafluoroethylene vessel, and cured after coating the $\mathrm{Cu}$-based mold. The mold-pattered PU membrane was then cut to match the $\mathrm{Cu}$-based negative template. The PDMS precursor (8:1 polymer:crosslinker) [35] was poured onto the $\mathrm{Cu}$-based template along one side, and left for $2 \mathrm{~h}$ to ensure complete wetting by the colloidal PDMS. The mold-patterned PU membrane was coated to prevent the PDMS colloid diffusing. The PDMS precursor was cured at $50^{\circ} \mathrm{C}$ for $4 \mathrm{~h}$, and removed carefully from the template to produce the primary setae array.

\subsection{Fabricating the hierarchical setae}

The $0.5 \mathrm{~g}$ PDMS colloid was poured onto a 3-inch planar $\mathrm{Si}$ surface for 5 min diffusion (Figure 1) and then placed under a vacuum with the Si template cleaned using Piranha solution (Figure 1(a)). After 10 min of pump (Edwards RV5, England) evacuation, the Si template was coated onto the planar Si surface. When the vacuum was relieved, the pressure of air forced the PDMS colloid into the Si template (Figure 1(b)). After removal from the template, the surface PDMS colloid, the Si template and the PDMS colloid were placed into an oven for curing (Figure 1(c)). After curing, the thin PDMS membrane was removed from the Si

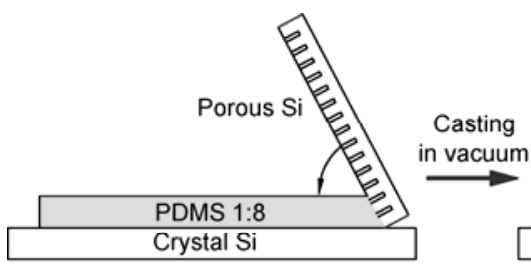

(a)

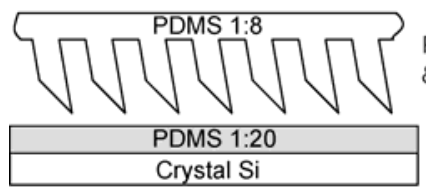

(e)

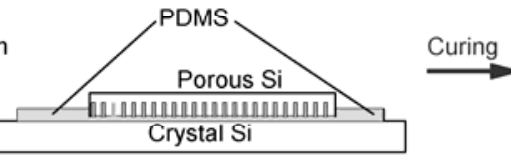

(b)

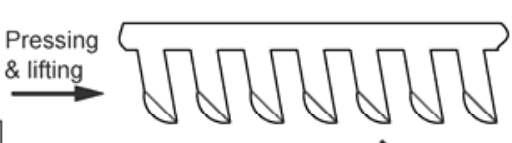

(f)
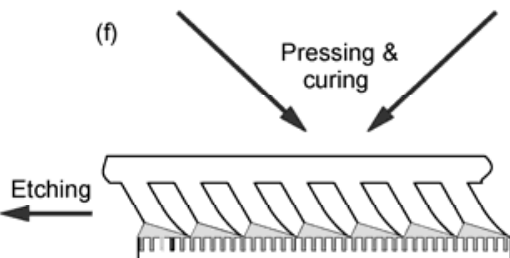

(g)

Figure 1 Schematic illustration of fabricating hierarchical micro-setae. 
template surface over a period of $48 \mathrm{~h}$ using plasma cleaner (PDC-VP2, USA) (Figure 1(d)). The secondary setae are formed inside Si template.

A PDMS colloid membrane (mass ratio 20:1) was coated onto another clean planar $\mathrm{Si}$ surface using spin-coating (KW-5, China) at $3000 \mathrm{r} / \mathrm{min}$ for $2 \mathrm{~min}$. The resultant primary setae (Section 2.2) were carefully placed onto this colloid membrane (Figure 1(e)), and removed after contacting the PDMS colloid, thus the tip of each seta has a small amount PDMS colloid on it (Figure 1(f)). By pressing this setae array onto the surface (Figure 1(d)) using a specially designed clamp (Figure $1(\mathrm{~g})$ ), after curing for $4 \mathrm{~h}$ at $50^{\circ} \mathrm{C}$, the secondary setae were firmly bonded on the ends of the primary setae. The Si template was etched with a solution of $\mathrm{HF}(40 \%): \mathrm{HNO}_{3}$ (concentrated) (volume ratio of 1:1) for 3 min at room temperature, thus forming a double-level setae array (Figure 1(f)).

\subsection{Observing morphologies}

The morphologies of the primary setae were observed by 3D optical microscopy (Keyence, VHX-600). The topographical structures of the $\mathrm{Si}$ template and hierarchical morphologies of the setae were observed by scanning electron microscopy (SEM) (LEO-1530VP, Germany) by using a Schottky field emission source. The setae samples were sputtered with gold particles $(\sim 10 \mathrm{~nm})$ prior to SEM observations.

\subsection{Testing adhesion performances}

All the adhesion experiments were done using a flat-on-flat tribometer (UMT-2, CETR, USA). A polished crystalline Si plate $(12 \mathrm{~mm} \times 25 \mathrm{~mm})$ was used as the upper mating plane, setae samples $(6 \mathrm{~mm} \times 8 \mathrm{~mm})$, containing 20 primary setae $(4 \times 5)$ were mounted on the substrate table. A complete measuring cycle comprises three stages: loading, slipping, and off-loading. During the loading stage, the upper sample approached the setae surface at a constant speed of 0.1 $\mathrm{mm} / \mathrm{s}$, and was kept in contact for $10 \mathrm{~s}$. During the slipping stage, the upper sample was moved forwards, backwards, and at an inclined angle $\left(8^{\circ}-10^{\circ}\right)$ under preloads varying from 0.5 to $25 \mathrm{mN}$. After the slipping stage, the upper sample was lifted, and the preload was decreased to zero. The data on the adhesive properties were collected by computer. Three measurements were taken to calculate a mean value. Three hierarchical setae arrays, derived the nature of the secondary setae were identified (1) radius $15 \mu \mathrm{m}$, pitch 30 $\mu \mathrm{m}$, (2) radius $10 \mu \mathrm{m}$, pitch $30 \mu \mathrm{m}$, and (3) radius $5 \mu \mathrm{m}$, pitch $20 \mu \mathrm{m}$.

\section{Results and discussion}

\subsection{Characterizations of the primary setae}

A 3D optical microscope can integrate images taken at var- ying depths into one 3D image by using embedding software-confocal microscopy. Figure 2 shows well defined 3D optical images of primary setae. Similarly to $\mathrm{Cu}$-based template, the seta radius is a nominal $0.5 \mathrm{~mm}$, with a mean height of $3.2 \mathrm{~mm}$. The setae have relatively even ends necessary if a uniform hierarchical seta array is to be fabricated. Each seta end is inclined at an angle of $45^{\circ}$ to the supporting surface, and at an angle of $30^{\circ}$ to the long axis of the seta rod. The rod is inclined at an angle of $75^{\circ}$ to the supporting surface, which is consistent with angle of $60^{\circ}-75^{\circ}$ observed on the gecko $[29,30]$.

\subsection{Characterizations of the hierarchical setae}

Compared to the $\mathrm{Cu}$-based template, the $\mathrm{Si}$-based template exhibits much finer topographical detail, which is below the resolution of an optical microscope, necessitating the use of SEM. As an example, plan view and cross-sectional SEM images of template for sample 1 are shown in Figure 3(a) and (b). A large number of regular pores can be seen in the plan view (Figure 3(a)), with radii of $15 \mu \mathrm{m}$, and a pitch of $30 \mu \mathrm{m}$. In Figure 3(b), the pore depths are nearly identical, at around $30 \mu \mathrm{m}$, but there is greater variation in the pore radii due to inherent process variation in the plasma etching.

Figure 3(c) and (d) presented the plan view SEM images of sample 1. In Figure 3(c), one double-level seta is clearly shown. Except for some upright secondary setae, many secondary setae have collapsed and buckled because the strong capillary tension generated during the process of drying, which causes adjacent micro-setae to buckle. Another cause of collapse is the effect of impact of gold particles $(10 \mathrm{~nm})$ during sputter coating. The substrate mounting of the setae sample was rotated to obtain clear SEM images of the morphologies of the secondary setae due to the inclination of the seta rods. In Figure 3(d), the setae have a mean radius of $13 \mu \mathrm{m}$, which is slightly less than that of Si-template; the mean pitch is around $39 \mu \mathrm{m}$, which is slightly more than that of Si-template. These changes probably come from the curing and mold unloading processes. During curing, $\mathrm{H}_{2}$ gas may be released resulting from the cross-linking reaction, which will inevitably occupy some space inside the Si template. During demolding, the PDMS elastomer may be slightly corroded by the etching solution.

To avoid detachment of the secondary setae, strong bonding is necessary. According to the supplier (Dow
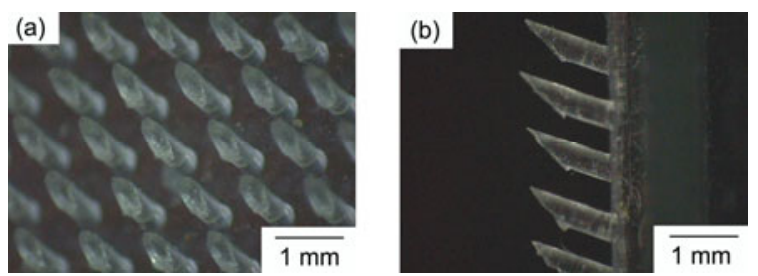

Figure 2 Optical images of the primary setae (a) top-view; (b) crosssectional view. 

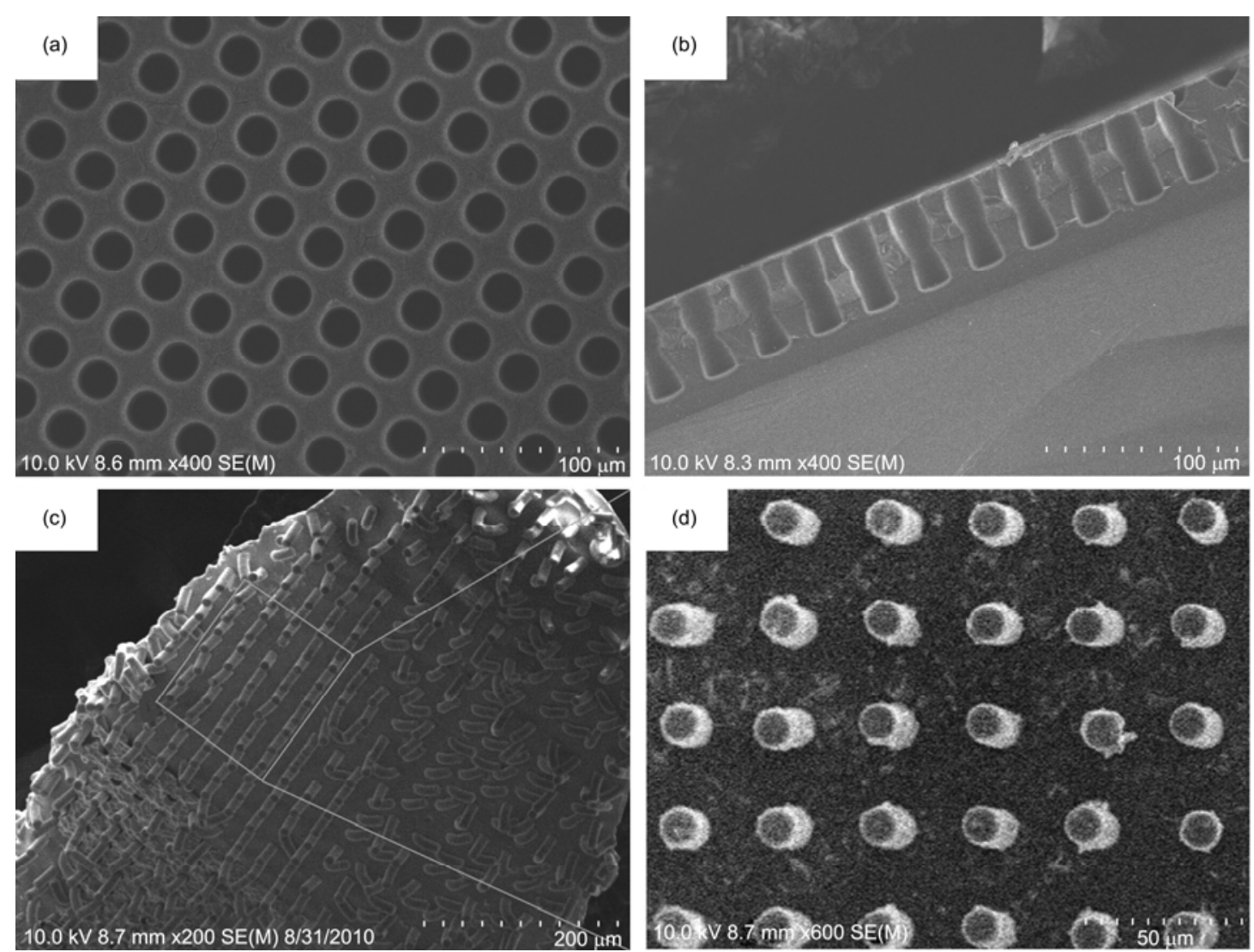

Figure 3 SEM images of the secondary setae and their templates. Top-view (a) and cross-sectional view (b) of Si template; top-view images of the double-level setae leaning $75^{\circ}$ to supporting surface (c) and the secondary setae leaning $45^{\circ}$ to supporting surface (d).

Corning), the main content of polymer is polymethylvinylsiloxane (PMVS), and the crosslinker is polymethylhydrosiloxane (PMHS). The usual weight ratio for the components is 10:1, and since PMVS and PMHS have equal active functions, the cross-linking reaction can proceed to completion. Typical hydrosilylation is shown in Figure 4.

When the ratio is greater than $10: 1$, for example $20: 1$, after curing, excess vinyl groups remain in the elastomer. Conversely, when the ratio is less than $1: 10$, for example $8: 1$, the silicone hydride group is in excess and residues remain after curing. Due to a high rate of diffusivity, silicone molecules can easily enter the PDMS membrane by thermal diffusion [35]. Thus the diffused silicone molecules can take the hydrosilylation reaction and the associated active functions inside PDMS membrane, thus improving the mechanical properties of the PDMS elastomer [35]. Therefore, the vinyl rich PDMS colloid from the 20:1 formulation can take part in hydrosilylation with the $\mathrm{Si}-\mathrm{H}$ rich
PDMS precursor from the 8:1 formulation, bonding the primary and the secondary setae. To avoid a "welded" structure, the thickness of the PDMS colloid (20:1) should be kept to a minimum.

The formulation of the etching solution plays an important role, because pure HF may not dissolve the crystalline Si template, whilst an etching solution with a high $\mathrm{HNO}_{3}$ concentration may corrode the PDMS elastomer. We have determined that a volume ratio of $1: 1\left(\mathrm{HF}: \mathrm{HNO}_{3}\right)$ is optimal, and that an etching time of around $3 \mathrm{~min}$ is required. The etching is finished once the black crystalline $\mathrm{Si}$ is completely gone.

\subsection{Determinations of adhesions}

The degree of adhesion is influenced by slipping distance, so a $2 \mathrm{D}$ force sensor was used to collect the adhesion data over a slipping distance of $3 \mathrm{~mm}$ under preloads of $0.5-25 \mathrm{mN}$.

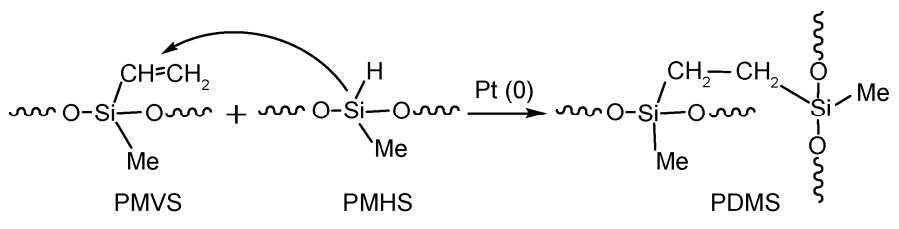

Figure 4 Typical reaction equation of PMVS and PMHS. 
From our measurements, maximum shear and normal adhesion are generated at preloads of $13 \mathrm{mN}$. Therefore, adhesion data from under $13 \mathrm{mN}$ is shown in Figure 5. To evaluate the adhesive strength, we adopted the area of the primary setae to act as the apparent contact area. Since each individual seta has an area of $0.464 \mathrm{~mm}^{2}$, the total area of 20 primary setae is $9.28 \mathrm{~mm}^{2}$. Considering radius and pitch, the absolute contact area of samples $1-3$ were $1.86,0.85$, $0.46 \mathrm{~mm}^{2}$. The maximum adhesive strengths are listed in Table 1.

In Figure 5, all the shear and normal adhesion curves exhibited show similar trends, initially rapidly increasing up to a slipping distance of $1.0 \mathrm{~mm}$, followed by a slight decrease at a slipping distance of $1.0 \mathrm{~mm}$, finally reaching a constant value when the slipping distance is greater than $1.2 \mathrm{~mm}$. The shear adhesions vary significantly between samples. Maximum shear adhesion appears at $1.0 \mathrm{~mm}$, with values of $51.08,38.86$, and $27.41 \mathrm{mN}$ for samples $1-3$. The corresponding maximum shear adhesion strengths are 2.75, 4.57, and $5.96 \mathrm{~N} / \mathrm{cm}^{2}$, and the stable shear adhesion strengths are $1.71,2.76$, and $3.20 \mathrm{~N} / \mathrm{cm}^{2}$.

As with shear adhesion, the maximum normal adhesions appear at a slipping distance of $1.0 \mathrm{~mm}$. Therefore, both shear and normal adhesion demonstrate the "slipping adhesion" effect, which is also observed in geckos [2]. Unlike shear adhesion, the adhesions of the seta arrays measured in the normal direction do not vary much. From Figure 5(b) and Table 1, all the maximum normal adhesion forces are about $10.8 \mathrm{mN}$, whereas the corresponding shear adhesion strengths are $0.58,1.27$, and $2.35 \mathrm{~N} / \mathrm{cm}^{2}$, and the stable adhesion strengths are $0.44,0.97$, and $1.79 \mathrm{~N} / \mathrm{cm}^{2}$.

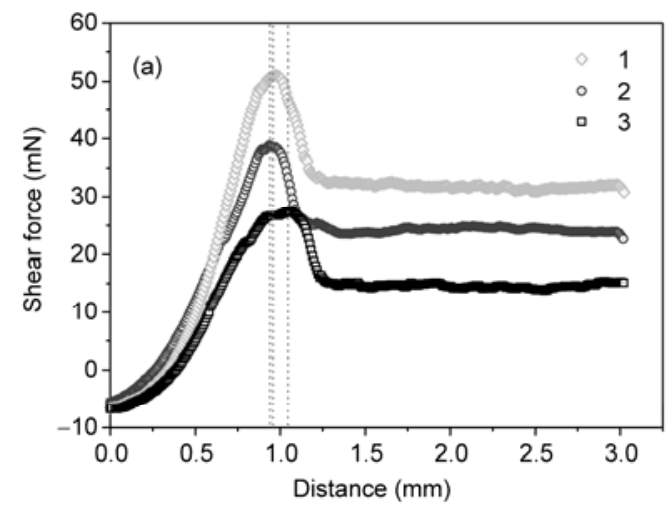

\subsection{Evolutions of shear adhesions}

Since the normal adhesions are relatively weak, and show little variation, the variation of shear adhesion with preload is significant. Additionally, as the primary setae are inclined at an angle of $75^{\circ}$ to the supporting surface, adhesion is influenced by the direction of motion. Therefore, forward, backward, and inclined movements were adopted to evaluate the adhesion performances of the seta arrays under varying preload, and those results are shown in Figure 6.

In Figure 6(a)-(c), when the upper sample is moved in the forward and inclined directions, the generated shear adhesions have similar trends. Adhesion increases with increasing preload between $0.5-13 \mathrm{mN}$, and the maximum adhesion is at $13 \mathrm{mN}$. When the preload is greater than 13 $\mathrm{mN}$, the adhesion gradually decreases. When the upper sample is moved backwards, the generated shear adhesion increases with increasing preload. As an example, the maximum adhesion of sample 1 is $50.51 \mathrm{mN}$ at a preload of $13 \mathrm{mN}$ for forward motion, $45.50 \mathrm{mN}$ at a preload of $13 \mathrm{mN}$ for inclined motion, and $10.27 \mathrm{mN}$ at a preload of $25 \mathrm{mN}$ or backward motion. The adhesion strengths are 2.72, 2.45, and $0.55 \mathrm{~N} / \mathrm{cm}^{2}$, respectively. The adhesion forces of for fward and inclined motions are similar at 4.9 and 4.4 times greater than for backward motion. These significant differences are related to the direction of motion. Forward motion is in the direction of inclination, so the contact area is stable, thus generating strong adhesion. For inclined motion, this is no longer the case and adhesion is slightly decreased. In backward motion, adhesion is significantly decreased because motion opposes the direction of inclination. Therefore,

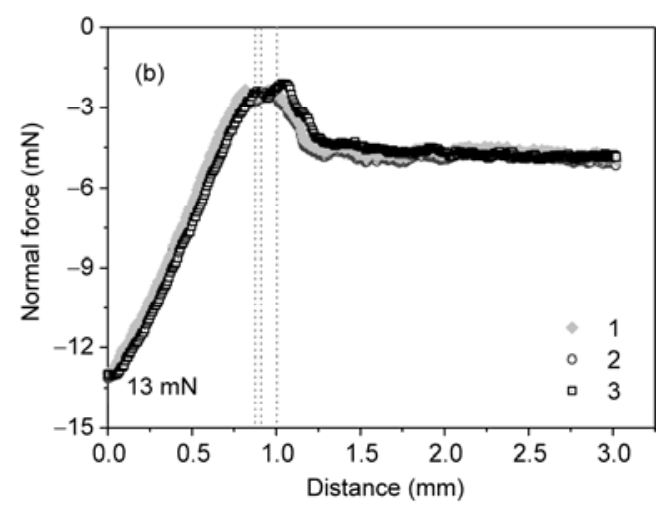

Figure 5 Slipping adhesion curves of sample 1-3 under the preload of $13 \mathrm{mN}$ (a) shear adhesion; (b) normal adhesion.

Table 1 Adhesion data of the sample 1-3 under the preload of $13 \mathrm{mN}$

\begin{tabular}{|c|c|c|c|c|c|c|c|c|c|}
\hline \multirow[t]{2}{*}{ Sample } & \multirow{2}{*}{$\begin{array}{l}\text { Contact area } \\
\qquad\left(\mathrm{mm}^{2}\right)\end{array}$} & \multicolumn{3}{|c|}{$\begin{array}{l}\text { Maximum shear adhesion } \\
(\mathrm{mN})\end{array}$} & \multicolumn{3}{|c|}{$\begin{array}{l}\text { Maximum shear adhesion strength } \\
\qquad\left(\mathrm{N} / \mathrm{cm}^{2}\right)\end{array}$} & \multirow{2}{*}{$\begin{array}{l}\text { Maximum normal } \\
\text { adhesion }(\mathrm{mN})\end{array}$} & \multirow{2}{*}{$\begin{array}{l}\text { Maximum norma } \\
\text { adhesion strength } \\
\left(\mathrm{N} / \mathrm{cm}^{2}\right)\end{array}$} \\
\hline & & Forward & Inclined & Backward & Forward & Inclined & Backward & & \\
\hline 1 & 1.86 & 51.08 & 45.50 & 10.27 & 2.75 & 2.45 & 0.55 & 10.8 & 0.58 \\
\hline 2 & 0.85 & 38.86 & 34.39 & 9.25 & 4.57 & 4.05 & 1.09 & 10.8 & 1.27 \\
\hline 3 & 0.46 & 27.41 & 23.67 & 8.20 & 5.96 & 5.15 & 1.78 & 10.8 & 2.35 \\
\hline
\end{tabular}



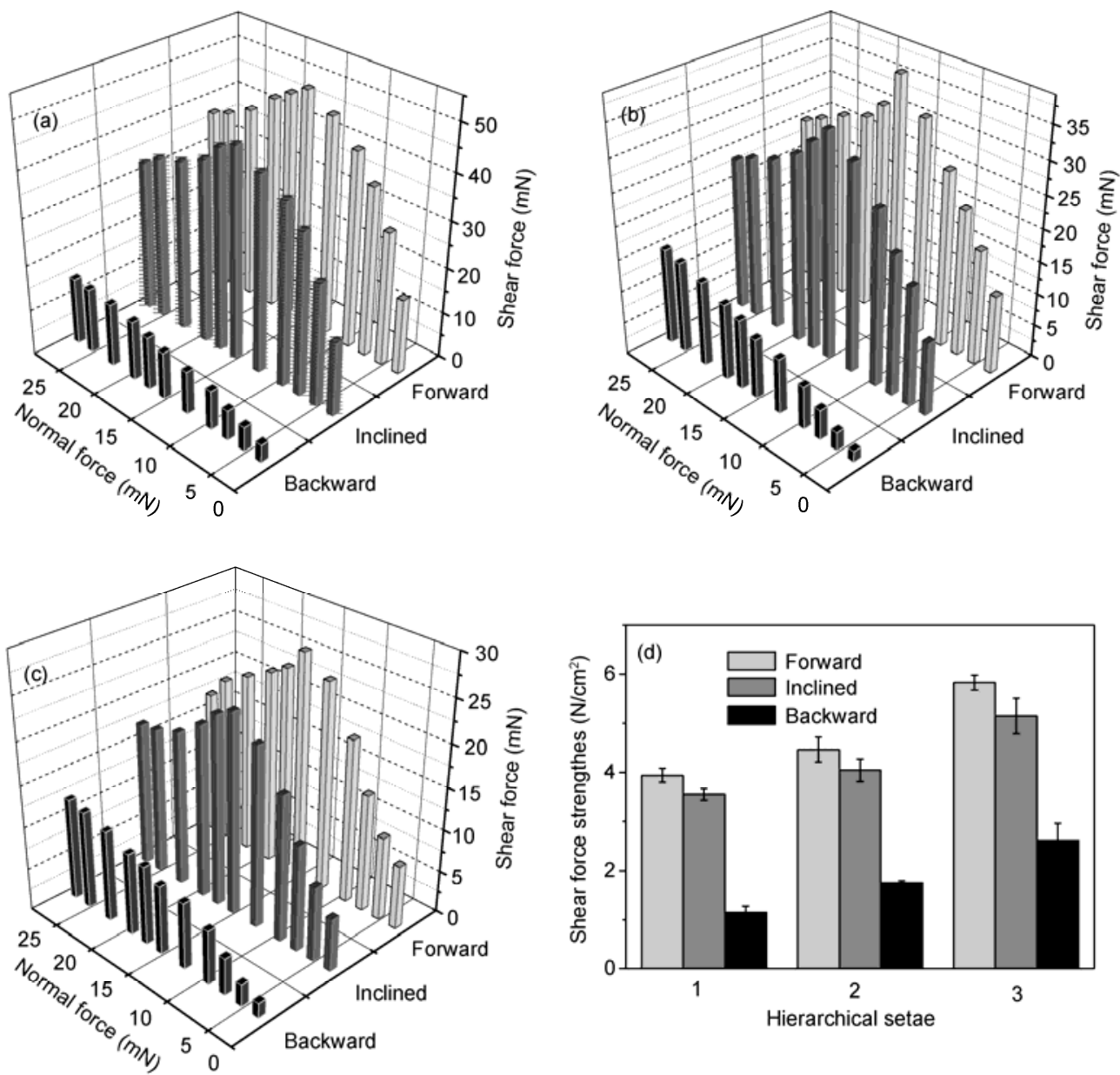

Figure 6 Comparisons of shear adhesions. (a) Sample 1; (b) sample 2; (c) sample 3; (d) column diagrams of the maximum adhesion strengths. Those mean deviations are $3.4,3.4,1.2 \% ; 5.6,5.6,2.2 \% ; 2.6,7.1$, and $14.9 \%$, respectively.

like the gecko, the resultant seta arrays have anisotropic adhesion.

The maximum shear adhesions of samples 2 and 3 are shown in Figure 6(b) and (c). For sample 2, the maximum adhesions are $38.86,34.39$, and $9.25 \mathrm{mN}$ in the three modes of motion. Forward is 4.2 times and inclined 3.7 times the magnitude of backwards. For sample 3, the maximum adhesions are $26.78,23.67$, and $12.10 \mathrm{mN}$. Forward is 2.2 times and inclined, 2.0 times the magnitude of backwards. These results are completely consistent with the JohnsonKendall-Robert (JKR) theory [36]. According to which, $F c=1.5 \pi R \gamma, F c$ represents the adhesion force, $R$ is the equivalent radius, $\gamma$ represents the equivalent surface energy. Due to the constitution the PDMS being kept constant, the change of adhesion mainly results from change in contact area. From Table 1, the contact area of sample 1 is the largest, which is 2.18 times of sample 2, and 4.26 times of sample 3 .

The maximum adhesion strengths of samples 1-3 under three modes of motion are shown in Figure 6(d). The adhesion strengths measured under forward motion are larger than those of inclined motion, which in turn are larger than those of backward motion. For the secondary setae samples, the adhesion strengths of sample 3 are larger than those of sample 2, which are larger than those of sample 1. These results are consistent with the "contact splitting" principle [37]. If one spherical large contact area is subdivided into $n$ smaller contacts, with an identical apparent contact area, the adhesion force rises by a factor $n^{1 / 2}$. Therefore, an array of setae with a small radius will generate strong adhesion. This is because the flexibility of a seta is closely related to its radius, with a seta with a small radius having a high degree of flexibility, allowing a larger effective contact area and hence, adhesion. Additionally, as the radius, pitch, and aspect-ratio are varying, the resultant setae arrays exhibit different collapse and self-buckling properties [38], with considerable influence on absolute contact areas. To optimize a seta array for adhesion performance, ideally to match that of a gecko, more work is required.

\section{Conclusions}

By using advanced spark-erosion perforating technology and an argon ion plasma etching technique, we have fabri- 
cated sub-millimeter-scale $\mathrm{Cu}$-based and micro-scale $\mathrm{Si}$ templates. Three types of hierarchical gecko-inspired setae arrays were successfully prepared by casting using these templates. Adhesion test results demonstrated: (a) the shear adhesion significantly increases, then, slightly decreases, finally reaching a stable state with increasing slipping distance; (b) adhesion under forward motion are larger than those measured under inclined motion, which in turn are much larger than those of backward motion, demonstrating that the resultant seta arrays have anisotropic adhesion performance like a real gecko; (c) the normal adhesion linearly increases when the preload is less than $13 \mathrm{mN}$, and slightly decreases when the preload is more than $13 \mathrm{mN}$; (d) the adhesive performance of secondary setae are vary. Seta with a large end area generates a strong apparent adhesion force, whilst seta with the small end area generates the strong adhesion strength.

This work was supported by the National Natural Science Foundation of China (50805076, 60910007), Doctoral Fund of Ministry of Education of China (200802871043) and Science Research Foundations of Nanjing University of Aeronautics \& Astronautics (NS2010216, NS2012014).

1 Gao H J, Yao H. Shape insensitive optimal adhesion of nanoscale fibrillar structures. Proc Natl Acad Sci USA, 2004, 101: 7851-7856

2 Autumn K, Sitti M, Liang Y A. Evidence for van der Waals Adhesion in Gecko Setae. Proc Natl Acad Sci USA, 2002, 99: $12252-12256$

3 Gao H J, Wang X, Yao H M, et al. Mechanics of hierarchical adhesion structures of geckos. Mech Mater, 2005, 37: 275-285

4 Dai Z D, Sun J R. Yue D, et al. Morphology and contact mechanics influence adhesive characteristics of dung beetle's bristle and gecko's setae. Prog Nat Sci, 2007, 17: 1074-1081

$5 \mathrm{Yu} \mathrm{M}$, Ji A H, Dai Z D. Effect of microscale contact state of polyurethane surface on adhesion and friction. J Bionic Eng, 2006, 3: 87-91

6 Dai Z D, Sun J R. Progress in study on gecko locomotion and biomimetic gecko-robot. Prog Nat Sci, 2007, 17: 1-5

7 Hansen W, Autumn K. Evidence for self-cleaning in gecko setae. Proc Natl Acad Sci USA, 2005, 102: 385-389

8 Kim S, Sitti M. Biologically inspired polymer microfibers with spatulate tips as repeatable fibrillar adhesives. Appl Phys Lett, 2006, 89: 261911

9 Murphy M P, Aksak B, Sitti M. Adhesion and anisotropic friction enhancements of angled heterogeneous micro-fiber arrays with spherical and spatula tips. J Adhes Sci Technol, 2007, 12: 1281-1296

10 Aksak B, Murphy M P, Sitti M. Gecko inspired micro-fibrillar adhesives for wall climbing robots on micro/nanoscale rough surfaces. IEEE Int Conf Rob Autom, 2008, 5: 3058-3063

11 Michael P M, Burak A, Sitti M. Gecko-inspired directional and controllable adhesion. Small, 2009, 5: 170-175

12 Murphy M P, Kim S, Sitti M. Enhanced adhesion by gecko-inspired hierarchical fibrillar adhesives. ACS Appl Mater Interfaces, 2009, 1: 849-855

13 Jeong $\mathrm{H}$ E, Lee $\mathrm{S}$, Kim $\mathrm{P}$, et al. High aspect-ratio polymer nanostructures by tailored capillarity and adhesive force. Colloids Surf A, 2008, 313: 359-364

14 Jeong H E, Lee S, Kim J K, et al. Nanoengineered multiscale hierarchical structures with tailored wetting properties. Langmuir, 2006,
22: 1640-1645

15 Jeong H E, Kwak R, Kim J K, et al. Generation and self-replication of monolithic, dual-scale polymer structures by two-step capillary-force lithography. Small, 2008, 4: 1913-1918

16 Jeong $\mathrm{H} \mathrm{E}$, Lee $\mathrm{J} \mathrm{K}$, Kim $\mathrm{H} \mathrm{N}$, et al. A nontransferring dry adhesive with hierarchical polymer nanohairs. Proc Natl Acad Sci USA, 2009, 106: 5639-5644

17 Greiner C, Arzt E, Campo A. Hierarchical gecko-like adhesives. Adv Mater, 2009, 21: 479-482

18 Campo A, Greiner C. SU-8: A photoresist for high-aspect-ratio and 3D submicron lithography. J Micromech Microeng, 2007, 17: R8195

19 Northen M T, Turner K L. A batch fabricated biomimetic dry adhesive. J Nanotechnol, 2005, 16: 1159-1166

20 Northen M T, Turner K L. Meso-scale adhesion testing of integrated micro- and nano-scale structures. Sens Actuators A, 2006, 130: 583-587

21 Northen M T, Greiner C, Arzt E, et al. A gecko-inspired reversible adhesive. Adv Mater, 2008, 20: 3905-3909

22 Zhang Y, Lin C T, Yang S. Fabrication of hierarchical pillar arrays from thermoplastic and photosensitive SU-8. Small, 2010, 6: 768-775

23 Kustandi T S, Samper V D, Ng W S, et al. Fabrication of a gecko-like hierarchical fibril array using a bonded porous alumina template. J Micromech Microeng, 2007, 17: 75-81

24 Jiang L, Yao X, Li H X, et al. "Water strider" legs with a selfassembled coating of single-crystalline nanowires of an organic semiconductor. Adv Mater, 2010, 22: 376-379

25 Sun J R, Cheng H, Cong Q, et al. Bionic study on the dung beetle copris ochus motschulsky for reduction of soil and adhesion (in Chinese). Acta Biophys Sin, 2001, 17: 785-793

26 Wang H J, Mei T, Wang X H. Adhesion array design of a novel biomimetic gecko crawling robot (in Chinese). Robot, 2006, 28: 191-194

27 Sun J R, Guo C, Cheng H, et al. Comparison of the setae between the dung beetle copris ochus and the gecko and the effects of deformation on their functions (in Chinese). Acta Zoologica Sinica, 2005, 51: 761-767

28 Wu L W, Zhang H, Li J B, et al. Fabrication and adhesion test of secondary structure with large end (in Chinese). Robot, 2011, 33: 222-228

29 Liu B, Zhang H, Guo D J, et al. Design, fabrication and adhesion test of lean bio-inspired seta (in Chinese). Tribology, 2009, 29: 393-398

30 Zhang H, Wu L W, Guo D J, et al. Adhesion performance of the multi-components silicone-based secondary structure cohesive arrays with large end (in Chinese). Tribology, 2011, 31: 295-303

31 Zhang H, Guo D J, Dai Z D. Progress on gecko-inspired micro/nano-adhesion arrays. Chin Sci Bull, 2010, 55: 1843-1850

32 Yu M, Mo G D, Jia S X, et al. Geometrical analysis and fabrication for biomimetic gecko-seta array (in Chinese). Journal of Nanjing University of Aeronautics \& Astronautics, 2010, 42: 739-743

33 Autumn K, Dittmore A, Santos D, et al. Frictional adhesion: A new angle on gecko attachment. J Exp Biol, 2006, 209: 3569-3579

34 Tian Y, Pesika N, Zeng H B. Adhesion and friction in gecko toe attachment and detachment. Proc Natl Acad Sci USA, 2006, 103: 19320-19325

35 Guo D J, Xiao S J, Liu H B, et al. Diffusion of hydrosilanes from the control layer to the vinylsilane-rich flow membrane during the fabrication of microfluidic chips. Langmuir, 2005, 21: 10487-10491

36 Johnson K L, Kendall K, Roberts A D. Surface energy and the contact of elastic solids. Proc R Soc A, 1971, 324: 301-313

37 Arzt E, Gorb S, Spolenak R. From micro to nano contacts in biological attachment devices. Proc Natl Acad Sci USA, 2003, 100: 10603-10606

38 Chandra D, Yang S. Stability of high-aspect-ratio micropillar arrays against adhesive and capillary forces. Accounts Chem Res, 2010, 43: 1080-1091

Open Access This article is distributed under the terms of the Creative Commons Attribution License which permits any use, distribution, and reproduction in any medium, provided the original author(s) and source are credited. 